\title{
STRUCTURE OF A WATER-INSOLUBLE D-GLUCAN ISOLATED FROM A STREPTOCOCCAL ORGANISM*,†
}

\author{
SATOAKI SHiBATA, IRWIN J. GoldStein, \\ Department of Biological Chemistry, University of Michigan, Ann Arbor, Michigan 48109 (U S.A.)
}

AND JEROME J. KIRKLAND

Procter \& Gamble Company, Miami Valley Laboratories, P.O. Box 39175, Cincinnati, Ohio 45247 (U.S.A.)

(Received November 22nd, 1982; accepted for publication. January 12th, 1983)

\section{ABSTRACT}

The structure of the extracellular polysaccharide (water-insoluble D-glucan) from an anaerobic, Gram-positive coccus organism (Streptococcus) has been investigated. Acid hydrolysis of the methylated glucan yielded 2,3,4,6-tetra-, 2,3,4tri-, 2,4,6-tri, and 2,4-di- $O$-methyl-D-glucose in the molar ratios of 1.13:3.99:1.00:1.02, indicating that the D-glucan has a branched structure containint $(1 \rightarrow 6)$ - and $(1 \rightarrow 3)$ - $\alpha$-D-glucosidic bonds with an average repeating unit of seven sugar residues. The D-glucan-polyalcohol, derived by successive periodate oxidation and borohydride reduction, gave, on complete hydrolysis with acid, glycerol and D-glucose (molar ratio, 2.2:1.0). Methylation of the D-glucan-polyalcohol yielded, upon hydrolysis, 2,4,6-tri-, and 2,4-di-O-methyl-D-glucose (molar ratio, 1.0:1.1). Methylation of the D-glucan-polyalcohol following Smith degradation (mild acid hydrolysis) gave 2,4,6-tri- $O$-methyl-D-glucose as the principal hydrolysis product, in addition to a trace of 2,3,4,6-tetra- $O$-methyl-D-glucose.

\section{INTRODUCTION}

Dextrans are branched $\alpha$-D-glucans containing predominantly $(1 \rightarrow 6)-\alpha$-Dglucosidic bonds, in addition to varying proportions of $(1 \rightarrow 2)-,(1 \rightarrow 3)$ - and $(1 \rightarrow 4)$ $\alpha$-D-glucosidic residues. These polysaccharides are the extracellular products of several microorganisms, some of which inhabit the oral cavity. The structure and physical properties of these polymers have been studied extensively ${ }^{1}$. In this communication, we report on the structure of a water-insoluble $\alpha$-D-glucan, the product of a Streptococcal organism isolated from the caecum of a weanling male rat.

\footnotetext{
*Dedicated to Professor Elvin A. Kabat.

${ }^{+}$This research was supported, in part, by a Grant (GM-29470) from the National Institute of General Medicine, National Institutes of Health.
} 


\section{RESULTS AND DISCUSSION}

Methylation by the Hakomori procedure ${ }^{2}$ of the water-insoluble D-glucan, followed by hydrolysis and g.l.c. analysis, gave the pattern shown in Fig. $1 \AA$. From the identity and molar ratios of the cleavage fragments (Table I), it is evident tha1 the D-glucan is highly branched with an average repeating-unit of seven sugar residues.

The methylation data suggest that the gross structure of this water-insoluble D-glucan resembles that of the water-insoluble D-glucan ${ }^{3}$ of Streptococcus mutans

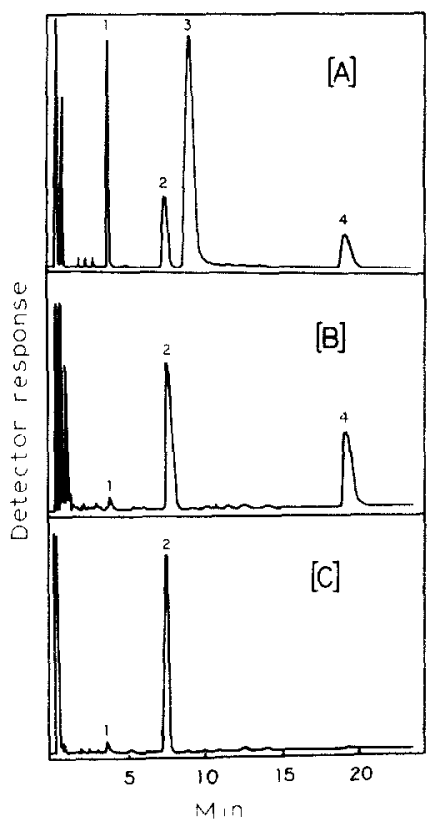

Fig 1 Gas-liquid chromatographic separation of the partially methylated alditol acetates from the Dglucan: (A) Natıve D-glucan. (B) products from D-glucan-polyalcohol, and (C) products from D-glucan derived from mild Smith degradation The peaks are: (1) 2,3,4.6-Tetra- $O$-methyl-D-glucone, (2) 2,4,6tri-O-methyl-D-glucose, (3) 2,3,4-tri-O-methyl-D-glucose and (4) 2,4-d1-O-methyl-D-glucose

\section{TABLE I}

MOLAR RAIIOS OF O-MFTHY C-D-GI UCOSES OBTAINED FROM HYDROLY LATES OF METHYLAIID IVSOI UBLF D-GI UCAV D-GLUCAN-POI YALCOHOI AVD PRODUCES HROM SMITH DEGRADAIIONOF D-GI UCAV

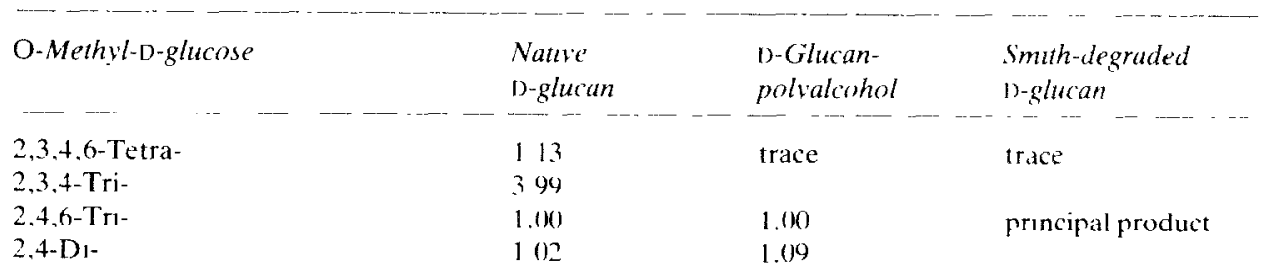




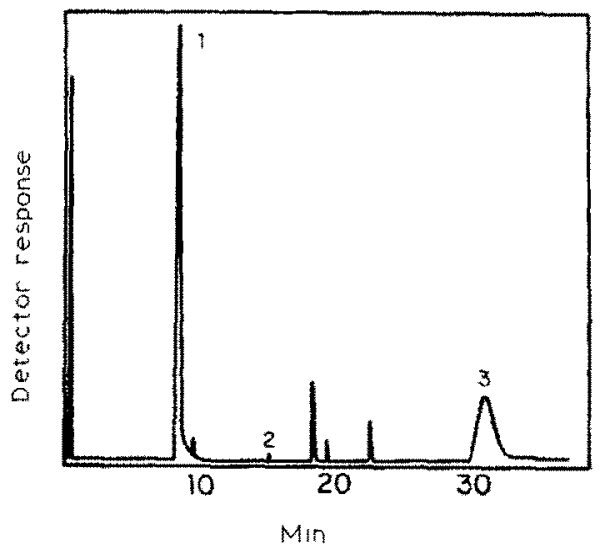

Fig. 2. Gas-liquid chromatographic separation of the alditol acetates of the products from complete Smith degradation. The peaks are: (1) Glycerol, (2) erythritol, and (3) D-glucitol.

OMZ 176, which has a branched structure (average repeating-unit, 7 sugar residues) consisting of $(1 \rightarrow 3)$ - and $(1 \rightarrow 6)$ - $\alpha$-D-glucosidic linkages in the ratio of $5: 2$; or dextran B-1355-S, which also has a branched structure (average repeating-unit, 10 sugar residues $\left.{ }^{4}\right)$ consisting of $(1 \rightarrow 3)$ - and $(1 \rightarrow 6)$ - $\alpha$-D-glucosidic bonds in the molar ratio of $4: 5$. However, the present D-glucan and that from Streptococcus mutans OMZ 176 are water-insoluble, whereas dextran B-1355-S is water-soluble.

Further structural information was obtained by subjecting the D-glucan to periodate oxidation. The periodate-oxidized polysaccharide was methylated, after reduction with sodium borohydride, hydrolyzed, and analyzed by g.l.c. (Table I and Fig. IB). The results confirmed that all $(1 \rightarrow 6)$-linked and terminal nonreducing D-glucose units were cleaved completely by periodate. When the D-glucan-polyalcohol was hydrolyzed by heating with $4 \mathrm{M}$ trifluoroacetic acid, glycerol and D-glucose were formed in the molar ratio of $2.2: 1$ (Fig. 2), as estimated by $\mathrm{g}$. I.c. This result is in good agreement with the methylation data. Hydrolysis of the D-glucan-polyalcohol with $50 \mathrm{~mm}$ sulfuric acid at $25^{\circ}$ (mild Smith degradation ${ }^{5}$ ) gave a water-insoluble, degraded D-glucan and, in the water soluble fraction, glycerol (identified by t.1.c.), which arose from nonreducing terminal D-glucose units and consecutive $(1 \rightarrow 6)$-linked D-glucosyl residues. The absence of any further water-soluble components, e.g., glucosylglycerol or glyceryl glycosides of gluco-oligosaccharides, indicated that the $(1 \rightarrow 3)$-linked D-glucose units comprise a block of periodate-resistant residues.

The results of the methylation of the Smith-degraded, water-insoluble D-glucan, followed by hydrolysis (see Table 1, Fig. 1C) indicate that the native D-glucan is highly branched and contains a backbone of consecutive $(1 \rightarrow 3)$-linked D-glucose residues. One possible structure for the native, water-insoluble D-glucan is illustrated by 1 . This represents a statistical or average repeating-unit.

The combined results of methylation analysis and Smith degradation also suggest that the structure of this D-glucan resembles that of Streptococcus mutans 


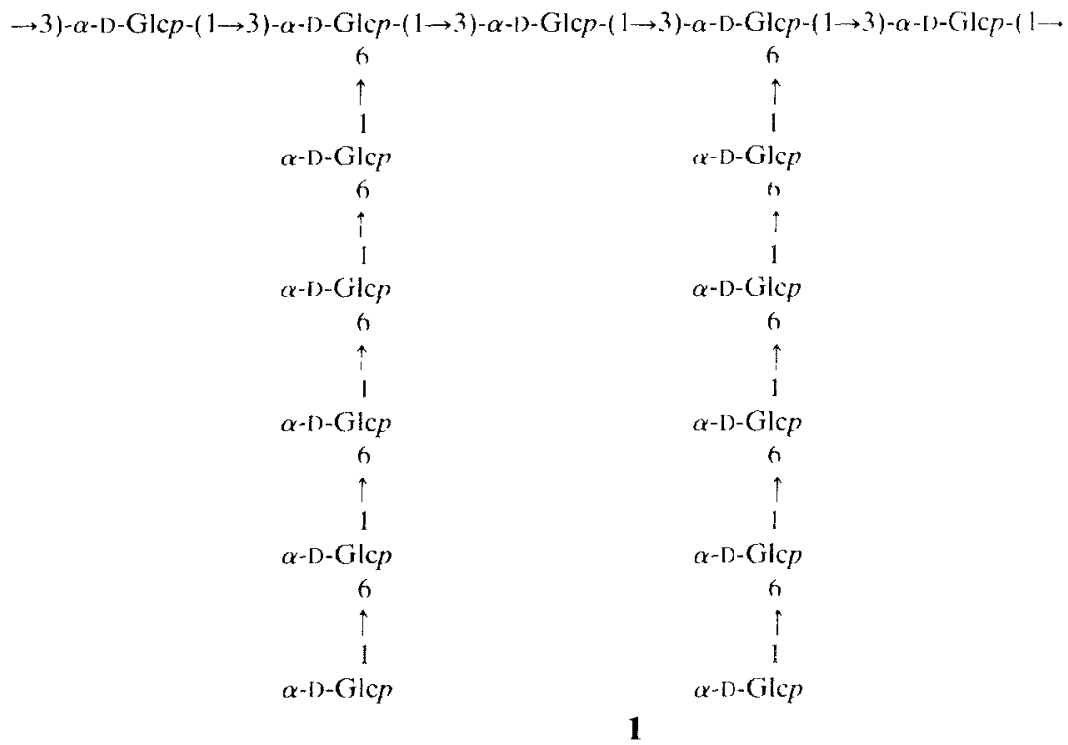

OMZ 176, which also possesses a backbone of consecutive $(1 \rightarrow 3)-\alpha$-linked Dglucose residues with short branches of $\alpha-(1 \rightarrow 6)$-linked D-glucose units as the side chains $^{3}$. Dextrans B-1355-S, which consists mainly of alternating $(1 \rightarrow 3)$ - and $(1 \rightarrow 6)-(x$-D-glucosidic linkages, is unlike these two water-insoluble D-glucans.

Additional evidence for the presence of consecutive $\alpha-(1 \rightarrow 6)$-linked D-glucose residues in the side chains was provided by the use of a dextranase from Penicillium $\mathrm{sp}$. When the native D-glucan was incubated with the dextranase from Penicillium sp., $\sim 60 \%$ of the D-glucose was liberated into the reaction medium as determined by the phenol-sulfuric acid method ${ }^{h}$.

The water-soluble products of the enzyme digest were subjected to gel filtra-

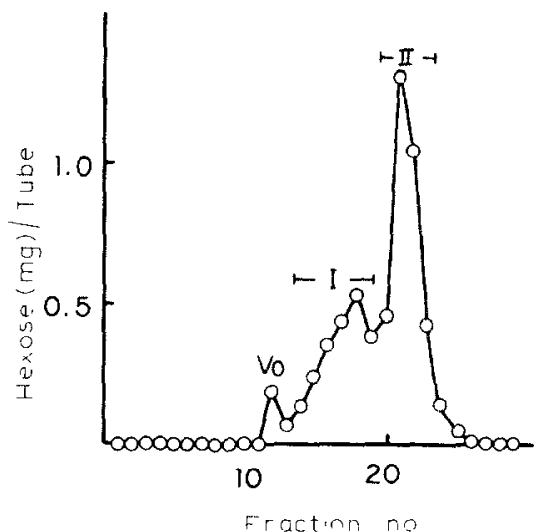

Fig. 3. Gel-filualion pofile of the Fencillum sp dexhandse digest of D-glucan on Bio-Gel P-2 columu $(1.5 \times 40 \mathrm{~cm}): 1-\mathrm{mL}$ fractions, $3 \mathrm{~mL} / \mathrm{h}$, and $V_{i 1}$, void volume 
tion (Bio-Gel P-2). Two major peaks (Peaks I and II) were obtained (Fig. 3). T.l.c. revealed that the principal components of Peak I were a tri- and tetra-saccharide; Peak II contained isomaltose with a trace of D-glucose. In model studies, the products of the action of the Penicillium sp. dextranase on a series of isomaltodextrins were shown to be mainly isomaltose with smaller proportions of D-glucose, and triand tetra-saccharides ${ }^{7}$. Thus, the dextranase-digestion data suggest that the side chains consist solely of $\alpha$-( $1 \rightarrow 6)$-linked D-glucose residues containing 4 or more units, whereas the polysaccharide core is composed of $(1 \rightarrow 3)$-linked D-glucose residues which are not attacked by the enzyme. This result is in good agreement with the methylation and periodate-oxidation data.

Analysis of the D-glucan by i.r. spectrometry gave a band at $840 \mathrm{~cm}^{-1}$ typical of $\alpha$-D-anomeric linkages, whereas no peak characteristic of $\beta$-D-glucosidic bonds was present at $890 \mathrm{~cm}^{-1}$. It would appear that the D-glucan consists solely of $\alpha$-Dglucosidic linkages.

A further indication of the $\alpha$-D-anomeric nature of the terminal nonreducing D-glucose groups is forthcoming from the interaction of the D-glucan with concanavalin A. Binding of the lectin was determined by allowing concanavalin A to react with the water-insoluble D-glucan and, after thorough washing of the complex, by displacement of the lectin with methyl $\alpha$-D-mannopyranoside (Fig. 4). It was determined that $16 \mu \mathrm{g}$ of concanavalin A were specifically bound to $1 \mathrm{mg}$ of polysaccharide. Inasmuch as the D-glucan contains no $\alpha-(1 \rightarrow 2)$ linkages, it may be concluded that the lectin binds to the polysaccharide chain-ends ${ }^{8}$.

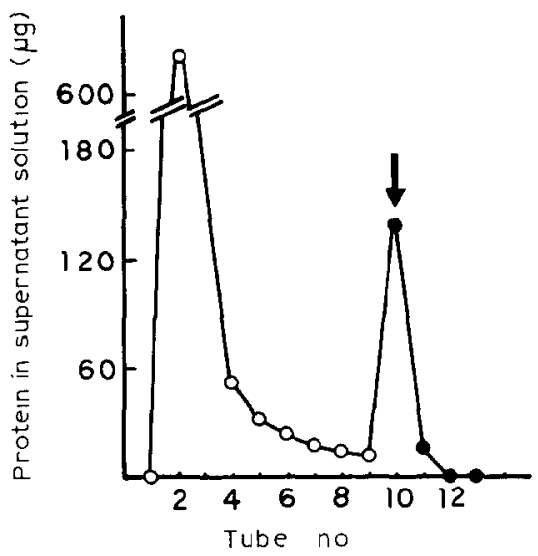

Fig. 4. The binding of concanavalin $\mathrm{A}$ to insoluble D-glucan. The binding and washing procedures are described in the Experimental section. (- - Protein concentration of the supernatant solution. (-) Protein concentration of the supernatant solution containing $50 \mathrm{mM}$ methyl $\alpha$-D-mannopyranoside. Arrow indicates wash number at which the mannoside was added. 
EXPERIMENTAL.

Materials. - The insoluble D-glucan from a Streptococcal organism was prepared as described hereafter. A dextranase preparation from Penicillium sp. was purchased from Sigma Chemical Co. (St. Louis, MO 43778), concanavalin A from Calbiochem-Behring Co. (San Diego, CA 92112), and Bio-Gel P-2 from Bio-Rad Laboratories (Richmond, CA 94804). Other sugars were available from previous studies, and all chemicals used were of reagent grade or the best quality available.

General methods. ... Protein concentration was determined by the Lowry et al. procedure" with bovine serum albumin as standard. Total carbohydrate content was determined colorimetrically by the phenol-sulfuric acid method ${ }^{5}$ with methyl $\alpha$-D-glucopyranoside as standard. T.I.c. was performed on precoated plates of Silica gel G-60) (Brinkmann Instruments. Inc.. Westburg. NV $1150(1)$ by the method of Hansen "'; the solvent system was 2:2:1 (viv) 2-propanol-acetone-M lactic acid. Carbohydrates were detected by spraying with a mixture of aniline (4 $\mathrm{mL})$, diphenylamine $(4 \mathrm{~g})$. acetone $(200 \mathrm{~mL})$, and $855^{\circ}$, phosphoric acid $(30 \mathrm{~mL})$, followed by heating the silica plates for $10 \mathrm{~min}$ at $140^{\circ}$. (i.l.c. was conducted in a Hewlett-Packard F \& M Scientific 412 High Efficiency gas chromatograph. equipped with a flame-ionization detector and a $\mathrm{U}$-shaped glass column $(0.4 \times 200 \mathrm{~cm})$. Methylated sugars were converted into their corresponding alditol acetates, and separated at $180^{\circ}$ on a column packed with $3 \%$ ECNSS-M on Gats-Chrom Q. For g.l.c. of Smith-degradation products, the acid hydrolyzate of the polyaccharidepolyalcohol was reduced with sodium borohydride, and acetylated by heding with $1: 1(\mathrm{v} / \mathrm{v})$ pyridine-acetic anhydride, for $1 \mathrm{~h}$ at 100$)^{\circ}$, and the product separated on the same column, at $80-19(\%$, programmed at a rate of $5 \%$ min; nutrogen was the carrier gas.

Isolation of polysaccharide. - The organism used in this study uas isolated from the caecum of a weanling male Charles River rat (Charles River Breeding Laboratories Inc., Wilmıngton. MA 01887) and is an anaerobic (iram-positive coccus. The organism has not been rigorously identified, but best fits the description of Streptococcus based on its hiochemical reactions and cellular morphology.

For isolation of extracellular polysaccharide, the organism wats grown in Trypticase Soy Broth (without D-glucose), BBL, and supplemented with 4.0 c's suc- $^{\circ}$ rose $(1 \mathrm{~L}$ ). The organism was grown for 5 days under anaerobic conditions (BBL GasPak Jars) at $37^{\circ}$. The cells were removed from the broth by centrifugation in the cold and the spent medium precipitated by $95^{\prime}$ " ethanol ( 3 vol.) at t wernight. The precipitate was collected and redissolved in sterile distilled water $(100) \mathrm{mL})$ The precipitation step was repeated two times. and the polysaccharide collected on a Millipore Filter, Type HA (Millipore Corp., Bedford, MA (11730), and dried with ethanol, followed with acetone. The extracellular polysaccharido was removed from the filter paer and stored in the freezer.

Methylation analysis. - Polysaccharides were methylated according to the method of Hakomori ${ }^{2}$. A dried sample $(10 \mathrm{mg})$ of the D-glucan was dissolved in di- 
methyl sulfoxide $(3 \mathrm{~mL})$ by ultrasonication for $5 \mathrm{~min}$ at $25^{\circ}$ in a nitrogen atmosphere, and methylated by treatment with methylsulfinyl carbanion $(1 \mathrm{~mL})$, followed by methyl iodide $(2 \mathrm{~mL})$. The mixture was dialyzed, concentrated to a small volume, and the methylated product extracted with chloroform. In the case of incomplete methylation, as judged by i.r. spectroscopy, the methylation procedure was repeated. The fully methylated polysaccharide was hydrolyzed by heating with $4 \mathrm{M}$ trifluoroacetic acid in a sealed tube for $5 \mathrm{~h}$ at $100^{\circ}$. The trifluoroacetic acid was evaporated and the methylated sugar components were reduced with sodium borohydride and converted into their corresponding alditol acetates by heating with $1: 1(\mathrm{v} / \mathrm{v})$ pyridine-acetic anhydride $(0.2 \mathrm{~mL})$ for $2 \mathrm{~h}$ at $100^{\circ}$, followed by analysis by g.l.c.

Periodate oxidation and Smith degradatin of the D-glucan; methylation of the degraded polysaccharide. - The native glucan $(30 \mathrm{mg})$ was oxidized with $50 \mathrm{~mm}$ sodium periodate $(20 \mathrm{~mL})$ at $5^{\circ}$ in the dark; at suitable time intervals, the periodate consumption was determined by the procedure of Avigad ${ }^{11}$. After oxidation for 7 days, excess periodate was decomposed by the addition of 1,2-ethanediol. The insoluble oxidation product was recovered by centrifugation, washed with distilled water, dissolved in $0.1 \mathrm{M}$ sodium hydroxide $(5 \mathrm{~mL})$, and reduced with sodium borohydride $\left(25 \mathrm{mg}\right.$ ); the mixture was kept overnight at $20^{\circ}$. Excess borohydride was decomposed by the addition of acetic acid (adjusting to $\mathrm{pH} 7.2$ ), the resulting reaction mixture containing insoluble D-glucan-polyalcohol dialyzed against distilled water at $4^{\circ}$, and the nondialyzable fraction lyophilized. A portion (2 mg) of the D-glucan-polyalcohol was hydrolyzed with $4 \mathrm{M}$ trifluoroacetic acid $(0.2 \mathrm{~mL})$ for $5 \mathrm{~h}$ at $100^{\circ}$. After evaporation of trifluoroacetic acid, the hydrolyzate was reduced with sodium borohydride, and the sugars were converted into the corresponding alditol acetates and analyzed by g.l.c. on a column of $3 \%$ ECNSS-M at $80-190^{\circ}$, programmed at the rate of $5 \% \mathrm{~min}$. The hydrolysis products were identified as glycerol and D-glucose, in the molar ratio of 2.2:1.0. Methylation of the D-glucan-polyalcohol (10 $\mathrm{mg}$ ) was achieved by the Hakomori procedure ${ }^{2}$. The methylated D-glucanpolyalcohol was hydrolyzed by heating with $4 \mathrm{M}$ trifluoroacetic acid for $5 \mathrm{~h}$ at $100^{\circ}$. The methylated sugar components were analyzed by g.l.c. as the corresponding alditol acetates (see Table I).

In another experiment, the D-glucan-polyalcohol $(10 \mathrm{mg})$ was subjected to Smith degradation by mild acid hydrolysis with $50 \mathrm{~mm}$ sulfuric acid $(2 \mathrm{~mL})$ for $18 \mathrm{~h}$ at $25^{\circ}$. The hydrolyzate was centrifuged to separate the insoluble, degraded polysaccharide from the water-soluble products. The supernatant solution was neutralized and examined by t.l.c. The insoluble D-glucan was washed with water and lyophilized. Following Hakomori methylation, the insoluble D-glucan was hydrolyzed and the methylated sugar components were analyzed by g.l.c. as the corresponding alditol acetates. (see Table I).

Degradation of the glycan by Penicillium sp. dextranase and characterization of the products. - The insoluble D-glucan $(10 \mathrm{mg})$ was incubated with dextranase from Penicillium sp. (Grade I, 20 units, Sigma) for 2 days at $37^{\circ}$ in a total volume 
of $2 \mathrm{~mL}$ of $0.1 \mathrm{M}$ phosphate buffer, $\mathrm{pH} 6.0$, containing $\mathrm{mm}$ manganese dichloride and a drop of toluene. After 2 days, the enzyme was inactivated by heating for 3 min at $85^{\circ}$, insoluble material was centrifuged off, and the enzyme digest, after concentration to $1 \mathrm{~mL}$, was subjected to gel-filtration on a column $(1.5 \times 40 \mathrm{~cm})$ of Bio-Gel P-2 (see Fig. 3). Peak tube fractions were analyzed by t.l.c.

Binding of concanavalin A to native D-glucan. - Binding studies were carried out in $3-\mathrm{mL}$ centrifuge tubes. Solutions were incubated for $48 \mathrm{~h}$ at $25^{\circ}$. The reaction mixture contained native, water-insoluble D-glucan $(9 \mathrm{mg})$ and concanavalin $A(300 \mu \mathrm{g})$ in a total volume of $0.9 \mathrm{~mL}$ of $0.15 \mathrm{~m}$ sodium chloride containing $0.1 \mathrm{M}$ of phosphate buffer, $\mathrm{pH} 7.2,0.1 \mathrm{~mm}$ calcium chloride, and $0.01 \%$ of sodium azide. After incubation for $48 \mathrm{~h}$ at $25^{\circ}$, the residual, insoluble D-glucan was centrifuged off in a refrigerated centrifuge $(8000 \mathrm{~g})$ for $20 \mathrm{~min}$ at $4^{\circ}$. and the supernatant solution was carefully decanted and saved. The recovered concanavalin A-Dglucan complex was washed several times by suspending in the same buffer $(0.9$ $\mathrm{mL}$ ), agitating with a Vortex mixer, centrifuging, and draining. Each supernatant (300- $\mu \mathrm{L}$ aliquots) was saved and the protein concentration determined by the Lowry et al. procedure ${ }^{9}$. When the total protein content of two successive supernatant solutions was less than $10 \mu \mathrm{g}$, the insoluble concanavalin A-D-glucan complex was washed 4 times with the same buffer containing $50 \mathrm{~mm}$ methyl $\alpha$-D-mannopyranoside, and the protein was determined.

\section{ACKNOWLEDGMENT}

The authors thank Dr. Robert Anderson for helpful discussions.

\section{REFERENCES}

1 R L. Sidebotha.M, Adv. Carbohydr. Chem Biochem., 30(1974) 371-44.4.

$2 \mathrm{~S}$ HAKOMORI, J Biochem. (Tokyo), 55 (1964) 205-208.

3 S. EBISL, A. Misaki, K. Kalo, ANDS KOIANi, Curbohydr Res, 22 (1972) 13-22.

4 A Misaki, M Torit, T. SaWai, and I J. Golostern, Carbohydr Res, 84 (1980) 273-285

5 I J Goldstein. G. W, Hay, B A. Lfwis. avd F Smith. Abutr Pap Am Chim Sol Meet 135 (1959) 3D.

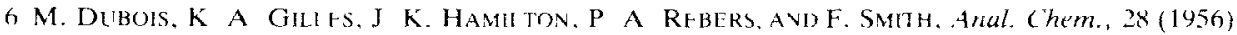
$350-356$.

7 E J Bourne, D. H Hutsov. avd H Weiget. Biochem. J, 85 (1962) 158-16.3

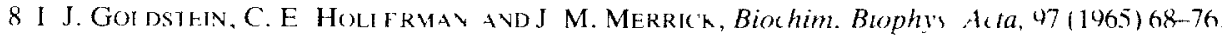

9 O. Iowry, N J. Rosfbrolich, A. L. Fakr and R I. Raydall, J. Blol (hem., 193 (1951) 26527.5

10 S A Havsen, J Chromatogr, 1015 (1975) 388-390

11 G. Avilgad, Carbohydr Res. 11 (1960) 119-123 